\title{
Chitosan Nanoparticles as a Percutaneous Drug Delivery System for Hydrocortisone
}

\author{
Haliza Katas, Zahid Hussain, and Tay Chai Ling \\ Drug Delivery and Novel Targeting Research Group, Faculty of Pharmacy, Universiti Kebangsaan Malaysia, \\ Kuala Lumpur Campus, Jalan Raja Muda Abdul Aziz, 50300 Kuala Lumpur, Malaysia \\ Correspondence should be addressed to Haliza Katas, haliz12@hotmail.com
}

Received 9 February 2012; Revised 23 March 2012; Accepted 26 March 2012

Academic Editor: Zhongkui Hong

Copyright ( $\odot 2012$ Haliza Katas et al. This is an open access article distributed under the Creative Commons Attribution License, which permits unrestricted use, distribution, and reproduction in any medium, provided the original work is properly cited.

Hydrocortisone (HC) has formed the mainstay for the management of atopic dermatitis. Hence, HC-loaded chitosan nanoparticles were prepared by ionic crosslinking of high, low molecular weight chitosan (HMwt, LMwt CS) and N-trimethyl chitosan (TMC) with tripolyphosphate. HC loading into CS nanoparticles was confirmed by FT-IR. The particle size of HC-loaded HMwt, LMwt, and TMC nanoparticles was increased from $243 \pm 12,147 \pm 11$, and $124 \pm 9 \mathrm{~nm}$ to $337 \pm 13,222 \pm 14$, and $195 \pm 7 \mathrm{~nm}$, respectively, by increasing the $\mathrm{pH}$ of CS solution. Their respective zeta potential and entrapment efficiency (EE) were significantly decreased by increasing the $\mathrm{pH}$ of CS solution. The swelling ratios of HC loaded HMwt, LMwt, and TMC NPs were increased when the pH of incubating media (PBS) was increased. The same increasing trend was observed in particle size and EE of HC loaded as the CS concentration was increased. The HC loaded CS NPs were generally nonspherical. In-vitro permeation studies showed that HC was efficiently released from the CS NPs in QV cream while in aqueous cream CS NPs provided a sustained release for HC. Thus, it is anticipated that CS NPs are the promising delivery system for anti-inflammatory drugs.

\section{Introduction}

Atopic dermatitis (AD) is a chronically relapsing, noncontagious and exudative dermatosis, associated with perivascular infiltration of IgE antibodies and $\mathrm{CD}_{4}$ lymphocytes [1]. AD is more prevalent during infancy and childhood. It is evident that the intense itching, dry scaly skin, and chronic inflammation are the symptoms commonly associated with $\mathrm{AD}$ [2]. Topical corticosteroids (TCs) have been recognized as the first-line pharmacological measure for the management of $\mathrm{AD}$ [3]. TCs provoke their pharmacological action by immunosuppressive, vasoconstrictive, anti-inflammatory and anti-proliferative effects [4]. In present study, hydrocortisone (HC) was used as the model drug due to its potential proficiency as topical agent since 1950s [5]. However, the chronic use of TCs was also associated with topical as well as systemic adverse effects $[6,7]$. However, in order to alleviate the TCs-associated deleterious effects and to rationalize the therapy, it is pertinent to use a suitable drug carrier system which can improve the rate and extent of drug permeation across the skin [8] and can improve the patient compliance.
Biodegradable polymeric nanoparticles (NPs) have attracted prominent interests in the past few decades as a novel drug carrier [9] due to its longer half life and greater drug entrapment efficiency [10]. Furthermore, the polymeric NPs embraced the site-specific targeting and tend to permeate deeply into the skin substructures [11] that are attributed to its nanoscale particle size $[12,13]$. Moreover, the biodegradable NPs can protect the drug from harsh environment [14] and prolong the duration of drug mucoadhesion at target tissues. Among all the recent materials used for polymeric NPs synthesis, chitosan (CS), a natural plentiful biopolymer obtained by chitin deacetylation has gained considerable interest. CS has promising biological implications such as nontoxic, biocompatible, biodegradable, bacteriostatic, and fungistatic $[15,16]$. In addition, CS also has strong mucoadhesive and adherence ability to conjugate with negatively charged sialic acid on the physiological membranes $[15,17]$. The main aim of the present study was to develop an occlusive and anti-inflammatory drug delivery system for the management of AD. 
In current research, CS NPs were successfully coloaded with $\mathrm{HC}$ by ionic crosslinking of CS with tripolyphosphate (TPP). The successful loading of HC into CS NPs was confirmed by FT-IR analysis. The physical characteristics (particle size, zeta potential, and EE) of HC-loaded CS NPs prepared at various preparation conditions such as different molecular weight, type, and concentration of CS were investigated. The swelling characteristics of $\mathrm{HMwt}$, LMwt, and TMC NPs were also studied at various $\mathrm{pH}$ of incubating media with a view to comprehend the release behavior of HC from CS NPs. Moreover, the in-vitro drug permeation study of HC from CS NPs either in aqueous or commercial QV cream was carried out at different $\mathrm{pH}$ of release media, using Franz diffusion cell. Incorporation of HC-loaded CS NPs into a commercial cream was carried out as to evaluate their versatility and simplicity in term of formulating them into various types of cream.

\section{Materials and Methods}

2.1. Materials. Low molecular weight (LMwt) CS (deacetylation degree (DD) $75-85 \%$ \& $70 \mathrm{kDa}$ ), high molecular weight (HMwt) CS (DD 85\%), hydrocortisone (98\%), and cellulose dialysis tubing (molecular cut-off of 12 to $14 \mathrm{kDa}$ ) were purchased from Sigma-Aldrich (USA). N-trimethyl chitosan (TMC) was obtained from Heppe Medical GmbH (Germany). Penta-sodium tripolyphosphate (TPP), disodium hydrogen phosphate, and methanol (HPLC grade) were sourced from Merck kGaA (Germany). Ethanol (95\%) was acquired from HmbG Chemicals (Selangor, Malaysia). Ego QV cream was purchased from Ego Pharmaceuticals PTY LTD (Malaysia). All other chemicals used were of analytical grade.

2.2. Preparation of CS NPs. CS NPs were prepared via ionicgelation method developed by Calvo et al. [18] with some modification. CS solutions $(2 \mathrm{mg} / \mathrm{mL})$ were prepared by dissolving HMwt and LMwt, CS in glacial acetic acid ( $1 \% \mathrm{v} / \mathrm{v})$ while, TMC was dissolved $(2 \mathrm{mg} / \mathrm{mL})$ in distilled water at room temperature. TPP solution $(1 \mathrm{mg} / \mathrm{mL})$ was prepared by dissolving it in distilled water. CS NPs were prepared spontaneously by adding $8 \mathrm{~mL}$ of TPP solution dropwise into $20 \mathrm{~mL}$ of CS solution, under a constant magnetic stirring at $700 \mathrm{rpm}$. Thereafter, CS NPs were centrifuged twice $(28$ $000 \mathrm{rpm}$ ) by using Optima L-100 XP Ultracentrifuge (Beckman-Coulter, USA) with a rotor NV 70.1 Ti (Beckman-Coulter, USA) for $30 \mathrm{~min}$ and lyophilized at $-40^{\circ} \mathrm{C}$ for $24 \mathrm{hrs}$.

2.3. Preparation of HC-Loaded CS NPs. For association of drug with CS NPs, the pure HC powder was dissolved into $30 \%$ ethanol with distilled water, to produce $1 \mathrm{mg} / \mathrm{mL}$ HC solution. Afterward, the HC solution was mixed with $0.2 \% \mathrm{w} / \mathrm{v}$ HMwt, LMwt or TMC CS at different $\mathrm{pHs}$ ranging from 3.0 to 7.0. To study the effect of CS concentrations, HC solution $(1 \mathrm{mg} / \mathrm{mL})$ was also mixed with various concentrations $(0.1,0.2,0.3,0.4,0.5 \% \mathrm{w} / \mathrm{v})$ of LMwt CS, and the mixtures were incubated for $15 \mathrm{~min}$ at room temperature. Then, $8 \mathrm{~mL}$ of TPP solution $(1 \mathrm{mg} / \mathrm{mL})$ was added dropwise into each reaction mixtures under magnetic stirring at $700 \mathrm{rpm}$, and HC-loaded CS NPs were simultaneously obtained. The nanoparticles were washed with distilled water and harvested by ultracentrifugation twice (Beckman-Coulter, USA) at $28000 \mathrm{rpm}$ and $10^{\circ} \mathrm{C}$ for $30 \mathrm{~min}$. The resulting pellets were lyophilized at $-40^{\circ} \mathrm{C}$ for $24 \mathrm{hrs}$ further analysis.

2.4. Particle Size and Zeta Potential of CS NPs. Mean particle size, polydispersity index (PDI), and zeta potential of CS NPs were measured by ZS-90 Zetasizer (Malvern Instruments, UK) which was based on the Photon Correlation Spectroscopy (PCS). Samples were dispersed in distilled water prior to measurement. All measurements were performed in triplicate at $25^{\circ} \mathrm{C}$ with a detection angle of $90^{\circ}$, and results were reported as mean \pm standard deviation.

2.5. Measurement of Entrapment Efficiency (EE\%) of HC. EE of $\mathrm{HC}$ was determined by measuring the ultraviolet (U.V) absorption of the respective supernatants of HC-loaded CS NPs obtained after ultracentrifugation. The corresponding calibration curves were made by subjecting the supernatants of standard HC solutions $(0.01,0.02,0.03,0.04,0.05,0.06$, $0.07,0.08,0.09,0.1 \% \mathrm{w} / \mathrm{v})$ under U.V/Vis spectrophotometer (U.V-1601; Shimadzu, Japan). The data obtained from U.V/ Vis spectrophotometer and reverse phase HPLC (Waters 600 controller, In-line Degasser AF, 2707 Autosampler, 2998 Photodiode Array Detector, USA) were compared. HC was measured at $248 \mathrm{~nm}\left(\lambda_{\max }\right)$. EE of $\mathrm{HC}$ was calculated according to the following formula [19]:

$$
\text { Entrapment efficiency }(\mathrm{EE})(\%)=\left(\frac{W_{t}-W_{f}}{W_{t}}\right) \times 100,
$$

where, $W_{t}$ is the total initial amount of $\mathrm{HC}$ and $W_{f}$ is the amount of free HC in the supernatant after ultracentrifugation. All measurements were performed in triplicate and results were reported as mean \pm standard deviation.

2.6. Swelling Study. The dry samples of HC-loaded HMwt, LMwt, and TMC NPs $(100 \mathrm{mg})$ were immersed in phosphate-buffered saline (PBS) at different pHs $(2,3,4,5,6,7$, and 8) and at room temperature for $24 \mathrm{hrs}$ until a swollen equilibrium was reached. The swollen samples were then collected by filtration, blotted with filter paper for the removal of the adsorbed water on the surface, and weighed immediately. The swelling ratio was calculated using the following formula [20]:

$$
\text { Swelling ratio }(\%)=\left(\frac{W_{s}-W_{d}}{W_{d}}\right) \times 100,
$$

where, $W_{s}$ and $W_{d}$ are the weights of swollen and dry samples, respectively. The results were reported as mean \pm standard deviations.

2.7. FT-IR Analysis. The FT-IR analysis of LMwt CS, pure HC, and HC-loaded LMW CS NPs were measured using a Fourier transform infrared spectrometer (Spectrum 100, 
Perkin Elmer, USA). Briefly, 2-3 mg of sample was mixed with $200-300 \mathrm{mg} \mathrm{KBr}$ and compressed to form transparent pellet. These pellets were scanned in transmission model. The FTIR spectra were recorded in the mid-IR region of $4000-400 \mathrm{~cm}^{-1}$ at resolution of $4 \mathrm{~cm}^{-1}$ with 32 coadded scans.

2.8. Morphological Analysis. Morphological characterization of HC-loaded CS NPs was carried out by using transmission electron microscopy (TEM). For TEM analysis, a drop of HC-loaded CS NPs dispersion was placed on the copper microgrid that was natively stained by phosphotungstic acid and evaporated at room temperature $\left(25 \pm 2^{\circ} \mathrm{C}\right)$ and then viewed under the electron microscope.

2.9. In-Vitro Drug Release Kinetics. In-vitro drug release profile of HC-loaded LMwt CS NPs $(0.2 \%$ w/v CS \& $0.1 \%$ TPP w/v) was carried out using a Franz diffusion cell. To study the rate and extent of $\mathrm{HC}$ release, two different formulations (QV cream and aqueous cream) were compounded, and their release behavior was examined at different $\mathrm{pHs}$ of release media.

2.9.1. Compounding of QV Cream Containing Pure HC or HCLoaded CS NPs. For compounding $5 \mathrm{~g}$ of QV cream containing HC-loaded CS NPs, the commercial QV cream (Ego Pharmaceuticals PTY LTD, Malaysia) was melted at $50^{\circ} \mathrm{C}$. Subsequently, the CS NPs suspension containing HC-loaded CS NPs equivalent to $25 \mathrm{mg}$ of $\mathrm{HC}$ was blended into the molten cream and shaken until a homogenous dispersion was obtained. A $0.5 \% \mathrm{HC}$ cream (control positive) was compounded by the same protocol as above which $25 \mathrm{mg}$ of pure $\mathrm{HC}$ was added into $5 \mathrm{~g}$ of QV cream.

2.9.2. Compounding of Aqueous Cream Containing HC or HCLoaded CS NPs. For preparing $5 \mathrm{~g}$ of aqueous cream containing $0.5 \% \mathrm{HC}$ (control positive), the required amount of pure HC powder was dissolved in melted emulsifying ointment. The subsequent procedure was performed as specified in British pharmacopoeia (B.P, 2010). A $5 \mathrm{~g}$ of aqueous cream containing HC-loaded CS NPs was prepared by adding the CS NPs suspension equivalent to $25 \mathrm{mg}$ of $\mathrm{HC}$ into the molten emulsifying ointment, and remaining procedure was performed as specified in B.P, 2010.

2.9.3. Drug Permeation Kinetic. A Franz diffusion cell was used to monitor the release profile of HC from both formulations (QV cream and aqueous cream) containing either HC-loaded CS NPs or pure HC $0.5 \%$ (control positive). The receptor phase was PBS ( $\mathrm{pH} 7.4 \& 4.0$ ) containing $30 \%$ ethanol, thermostatically maintained at $37^{\circ} \mathrm{C}$ with circulating water. Dialysis membrane with molecular weight cut off of $12-14 \mathrm{kDa}$ was used to separate receptor and donor compartments. Thereafter, $1 \mathrm{~g}$ of each formulation (QV cream and aqueous cream) containing CS NPs or pure HC was placed into the donor compartment. After an appropriate time interval, $1 \mathrm{~mL}$ of sample was taken from the receptor phase and supplemented with an equal volume of fresh receptor medium. Diffusion of HC from the donor compartment was estimated spectrophotometrically at $248 \mathrm{~nm}$ $\left(\lambda_{\max }\right)$. The cumulative drug permeation percentage was determined by the following formula:

$$
\text { Cumulative permeation percentage }(\%)=\left(\frac{W_{t}}{W_{l}}\right) \times 100 \text {, }
$$

where, $W_{t}$ is the amount of HC released from the CS NPs at time $t$, and $W_{l}$ is the amount of HC initially loaded into the CS NPs.

2.10. Rheology Study. Rheology study was carried out to determine the apparent viscosity of QV cream and aqueous cream containing pure HC and HC-loaded CS NPs. The values of apparent viscosity of both the formulations were investigated before and after drug loading by using Bohlin Gemini Rheometer (U.K) with cone and plate system $1 \%$ $40 \mathrm{~mm}$. The applied shear rate was ranged from 0.005 to $300 \mathrm{~s}^{-1}$ and each test was run for $2 \mathrm{~min}$. All measurements were performed at $32^{\circ} \mathrm{C}$ with controlled shear rates. Each run was performed in triplicate, and the results were presented as mean \pm standard deviation.

2.11. Statistical Analysis. All the data were presented as mean \pm standard deviation (SD). Data were analyzed with either paired $t$-test or independent $t$-test and analysis of variance (One-way ANOVA) by using SPSS 19.0. For independent $t$ test, paired $t$-test, and ANOVA, $P<0.05$ showed significant difference between the tested groups.

\section{Results and Discussions}

3.1. Effects of $p H$ of CS Solution on the Characteristics of CS NPs. Physical characteristics (particle size, zeta potential, and EE) of HC-loaded CS NPs prepared from HMwt, LMwt, and TMC CS $(0.2 \%$ w/v CS \& $0.1 \%$ TPP w/v) were investigated at various $\mathrm{pH}$ of $\mathrm{CS}$ solution to comprehend the effects of molecular weight and $\mathrm{pH}$ of $\mathrm{CS}$ on their physical characteristics.

3.1.1. Particle Size and Zeta Potential. The mean particle size of HC-loaded CS NPs was influenced by the molecular weight and type of CS as well as pH of CS solution. When comparing two different molecular weights, a larger particle size of HC-loaded CS NPs was obtained with HMwt CS at various $\mathrm{pH}$ of CS solutions. As summarized in Figure 1(a), the mean particle size of HC-loaded HMwt CS NPs was significantly varied (ANOVA post-hoc tukey, $P<0.05$ ) from $243 \pm 12$ to $337 \pm 13 \mathrm{~nm}$ with varying $\mathrm{pH}$ of $\mathrm{CS}$ solution from 3 to 7. The mean particle size of LMwt and TMC was also significantly increased by increasing the $\mathrm{pH}$ of CS solution from pH 3.0 to 7.0 (one-way ANOVA post hoc tukey $P<0.05$ ). However, the mean particle size between LMwt and TMC at the same $\mathrm{pH}$ was not significantly different. The increasing trend in particle size with increasing $\mathrm{pH}$ could be due to the formation of agglomeration as repulsive forces between CS NPs decreased with increasing the $\mathrm{pH}$ of 


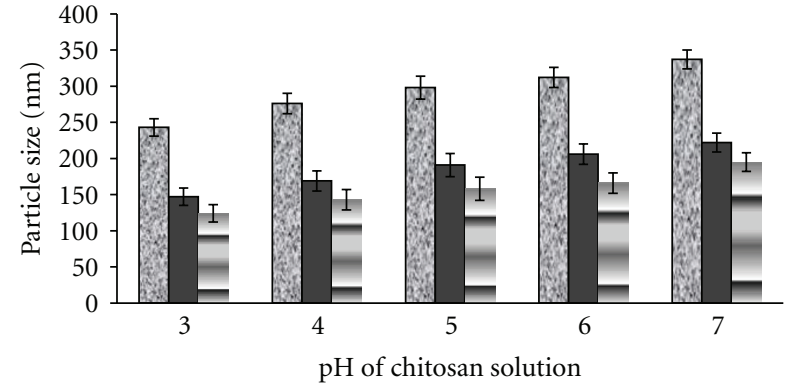

(a)

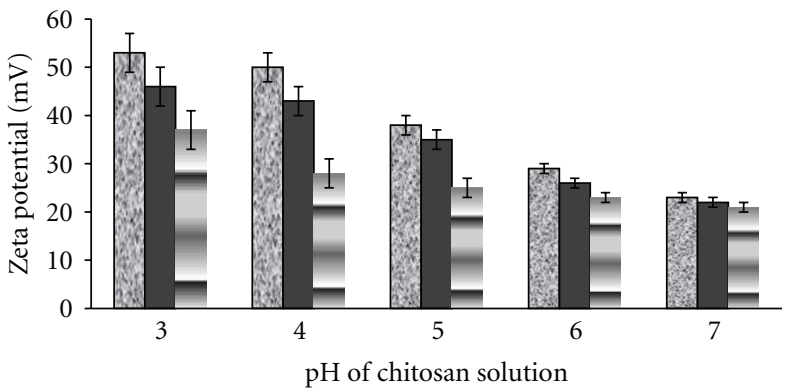

(b)

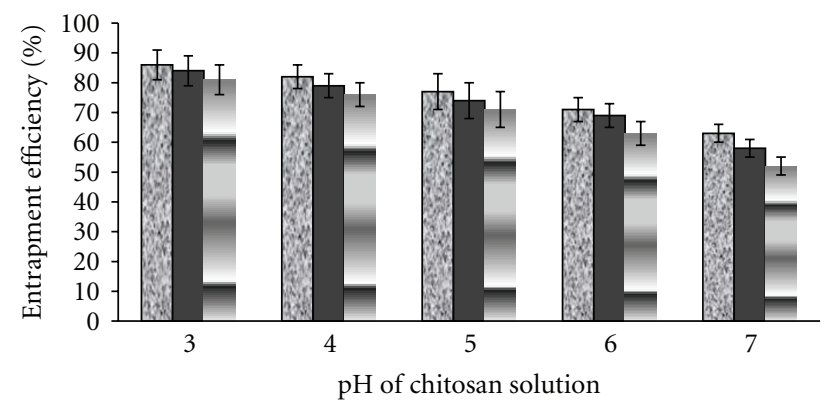

문 HMwt CS NPs

- LMwt CS NPs

$\equiv$ TMC NPs

(c)

Figure 1: Effects of pH of HMwt, LMwt CS, and TMC solutions on (a) particle size (b) zeta potential, and (c) entrapment efficiency (EE) of HC-loaded CS NPs (CS $0.2 \% \mathrm{w} / \mathrm{v}, \mathrm{HC} 1 \mathrm{mg} / \mathrm{mL}$, and TPP $1 \mathrm{mg} / \mathrm{mL}, n=3$ ).

CS solution [21]. The reduction in repulsive forces between particles was contributed by the decrease in the protonation of the $-\mathrm{NH}_{2}$ groups on the contour of CS. This was also corroborated with the findings that a considerable decrease in the zeta potential of HMwt, LMwt, and TMC CS NPs was observed when the $\mathrm{pH}$ of CS solution was increased as shown in Figure 1(b). Data also demonstrated that the zeta potential of HMwt CS NPs was remarkably decreased from $+53 \pm 6$ to $+23 \pm 3 \mathrm{mV}$ by increasing the $\mathrm{pH}$ from 3 to 7 . Similar to that, the zeta potential of LMwt CS and TMC NPs was reduced from $+46 \pm 5$ to $+22 \pm 3 \mathrm{mV}$ and $+37 \pm 3$ to $+21 \pm 2 \mathrm{mV}$, respectively, when the $\mathrm{pH}$ of CS solution was increased. As previously mentioned, decrease in the zeta potential could be explained by the reduction in the protonation extent of $-\mathrm{NH}_{2}$ groups on the CS backbone [21]. The mean value of the zeta potential of HC-loaded HMwt CS NPs was comparatively higher than the HC-loaded LMwt as well as TMC CS NPs at pH 4 and 6. This could be occurred as a higher molecular weight of CS contains higher density of positively charged $-\mathrm{NH}_{3}{ }^{+}$groups on the CS chain that is responsible to increase the surface charge of CS NPs. In addition to that, during the crosslinking process, TPP ions were counteracted by the positively charged $-\mathrm{NH}_{3}{ }^{+}$groups of HMwt CS which were quantitatively more than the phosphoric groups of TPP. However, less $-\mathrm{NH}_{3}{ }^{+}$groups were available for LMwt CS and TMC to counter balance the negatively charged phosphoric groups of TPP [22]. Furthermore, the derivatization of TMC may also decrease the $-\mathrm{NH}_{3}{ }^{+}$ groups on the backbone of TMC which rendered its low zeta potential. However, the differences among the mean value of the zeta potential of HC-loaded HMwt, LMwt CS, and TMC NPs were not statistically significant (one-way ANOVA post hoc tukey) at other pHs. Therefore, it could be concluded that different molecular weights and types of chitosan used in this study had no major impact on the surface charge of the NPs at $\mathrm{pH}$ other than $\mathrm{pH} 4$ and 6.

3.1.2. EE of HC-Loaded HMwt, LMwt and TMC NPs. The EE of $\mathrm{HC}$ decreased significantly (one-way ANOVA, $P<0.05$ ) from 86 to $63 \%$ for HMwt CS NPs with increasing the $\mathrm{pH}$ of CS solution as shown in Figure 1(c). This was expected to be due to the decreased positively charged $-\mathrm{NH}_{3}{ }^{+}$groups along the CS chain which therefore reduced the entanglement efficiency of CS with HC and decreased the EE. Besides, it was also observed that the EE of HC for HMwt, LMwt, and TMC NPs was not significantly different from each other over the range of $\mathrm{pH}$ used in the present study except at $\mathrm{pH}$ 7.0. These results were also corroborated with the previously reported findings. The findings showed that the EE of NPs prepared from different molecular weights of CS was not statistically different [23]. Furthermore, Vila et al. proposed that the entanglement of drug with CS is highly irrespective of the CS molecular weights and predominantly dependent on the degree of CS deacetylation [24]. 
TABLE 1: Particle size, zeta potential, PDI, and EE of HC-loaded LMwt CS NPs at various concentrations of CS (Mean \pm S.D, $n=3$ ).

\begin{tabular}{|c|c|c|c|c|c|}
\hline $\begin{array}{l}\text { CS concentration } \\
(\%)\end{array}$ & $\begin{array}{l}\text { Particle size } \\
(\mathrm{nm}) \pm \text { S.D }\end{array}$ & PDI & $\begin{array}{c}\text { Zeta potential } \\
(\mathrm{mV}) \pm \mathrm{S} . \mathrm{D}\end{array}$ & $\begin{array}{c}\mathrm{EE}(\%) \pm \text { S.D } \\
\text { HPLC }\end{array}$ & $\begin{array}{c}\mathrm{EE}(\%) \pm \mathrm{S} . \mathrm{D} \\
\text { U.V/VIS. }\end{array}$ \\
\hline 0.1 & $112 \pm 11$ & 0.31 & $+33 \pm 3$ & $86 \pm 3$ & $84 \pm 2$ \\
\hline 0.2 & $194 \pm 9$ & 0.24 & $+44 \pm 4$ & $88 \pm 3$ & $84 \pm 2$ \\
\hline 0.3 & $299 \pm 8$ & 0.38 & $+52 \pm 4$ & $88 \pm 4$ & $84 \pm 1$ \\
\hline 0.4 & $405 \pm 12$ & 0.41 & $+51 \pm 2$ & $89 \pm 4$ & $85 \pm 2$ \\
\hline 0.5 & $537 \pm 15$ & 0.47 & $+44 \pm 4$ & $90 \pm 5$ & $86 \pm 1$ \\
\hline
\end{tabular}

Besides, TMC NPs showed a decrease pattern in the mean value of HC EE from pH 3 to 7 as shown in Figure 1(c) which might be caused by the derivatization of amino groups $\left(-\mathrm{NH}_{2}\right)$ with ethyl groups $\left(-\mathrm{CH}_{3} \mathrm{CH}_{2}\right)$ along the CS-contour TMC.

3.2. Swelling Analysis. In order to investigate the effects of $\mathrm{pH}$ values on the physical behavior of HC-loaded HMwt, LMwt, and TMC NPs, a series of experiments were carried out. This study was performed at different $\mathrm{pH}$ of incubating media, ranging from $\mathrm{pH} 3$ to 7 as to determine physical behavior of HC-loaded CS NPs at physiological and pathological environments. $\mathrm{pH}$ of intact skin ranges from 4.8 to nearly 6.0. This condition is mainly due to the presence of "acid mantle". It acts as a natural skin barrier to the external environment. The wound site of AD patient would normally have a higher $\mathrm{pH}$ which ranging from neutral to alkaline due to the leakage of exudates and transudates (interstitial fluid) as well as disruption of "acid mantle". Thus, the knowledge regarding the impact of $\mathrm{pH}$ on the swelling ratio of various types of CS NPs is vital. It is known that the pKa value of CS is 6.5 [25]. At $\mathrm{pH} 2$, the swelling may be caused by the strong electrostatic repulsion between CS molecules as most of the amino groups of CS were in $-\mathrm{NH}_{3}{ }^{+}$form, the molecules therefore had net positive charge. Thus, it is probably assumed that the extended swelling conformation of CS at low range of $\mathrm{pH}$ could be caused by both the hydration of $-\mathrm{NH}_{3}{ }^{+}$groups as well as strong positive-positive charge repulsion among the $-\mathrm{NH}_{3}{ }^{+}$groups along the contour of CS. Contrary to that, at higher $\mathrm{pH}$ values, probably $3.0-5.0$, the amino groups in $\mathrm{CS}$ are less extensively protonated and the phosphoric groups of TPP are ionized; thus, the week electrostatic attractions produced between CS and TPP that restrain the swelling of CS NPs. Whereas, at further higher $\mathrm{pH}$ values $(\mathrm{pH}=6-8)$, most of the amine groups of CS and phosphoric groups of TPP were in the $-\mathrm{NH}_{2}$ and $-\mathrm{PO}_{4}{ }^{-}$form, respectively. As a result, the electrostatic attractions between CS and TPP became weakened which favor the swelling of CS NPs as shown in Figure 2. This pH-responsive characteristic of CS $\mathrm{NPs}$ can be used for the development of $\mathrm{pH}$-responsive drug delivery systems.

\subsection{Effect of CS Concentration on the Characteristics of CS NPs}

3.3.1. Particle Size and Zeta Potential. For this and the following experiments, CS NPs were prepared by ionic crosslinking

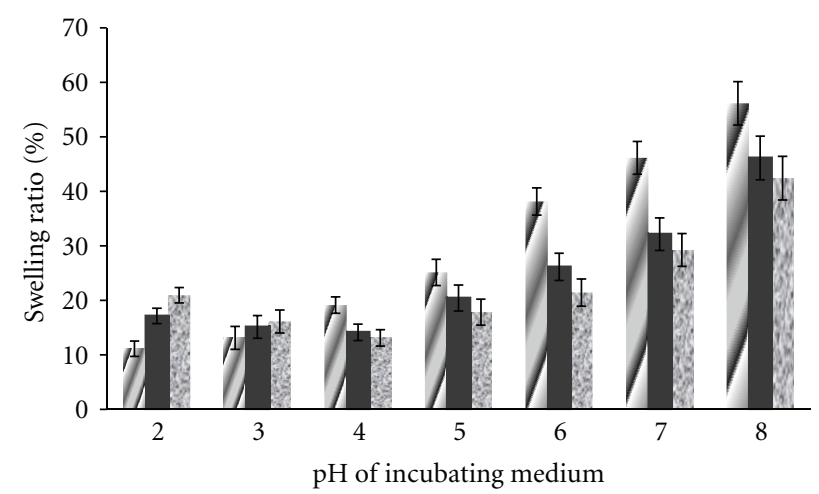

17. HMwt CS NPs
LMwt CS NPs
‥ TMC NPs

FIGURE 2: Effects of $\mathrm{pH}$ of PBS buffer solution on the swelling ratios of HC-loaded HMwt, LMwt, and TMC NPs (CS 0.2\% w/v, HC $1 \mathrm{mg} / \mathrm{mL}$, and TPP $1 \mathrm{mg} / \mathrm{mL}, n=3)$.

of LMwt CS with TPP. LMwt CS was chosen because it produced the desired size of nanoparticles with high EE. To investigate the effects of CS concentration on the physical characteristics of resultant CS NPs, various concentrations $(0.1,0.2,0.3,0.4$, and $0.5 \%)$ of LMwt CS were prepared at $\mathrm{pH} 3.0$ as to obtain HC-loaded CS NPs of nanosized and positively charged particle with the highest EE of HC. The data obtained (Table 1) revealed that the particle size of HCloaded LMwt CS NPs was significantly increased $(P<0.05)$ from $112 \pm 11$ to $537 \pm 15 \mathrm{~nm}$, with increasing the CS concentration from $0.1 \%$ to $0.5 \% \mathrm{w} / \mathrm{v}$. These results were in accordance with other reports $[18,26]$. The increase in particle size was expected to be due to the presence of intermolecular hydrogen bonding (due to $-\mathrm{OH}$ groups) and intermolecular electrostatic repulsion (due to $-\mathrm{NH}_{3}{ }^{+}$groups) which exist along the contour of CS [27]. As the concentration of CS increases, more molecules of CS tend to entangle with each other and crosslink with counter ion (TPP) to form a single larger particle [26].

The zeta potential of HC-loaded CS NPs was also increased from $+33 \pm 3$ to $+52 \pm 4 \mathrm{mV}$, when the CS concentration was increased from 0.1 to $0.3 \% \mathrm{w} / \mathrm{v}$. However, the surface charge of CS NPs decreased suddenly when LMwt CS concentration was increased from $0.4 \%$ to $0.5 \% \mathrm{w} / \mathrm{v}$ as shown in Table 1 . The increase in zeta potential from $0.1 \%$ 
to $0.3 \% \mathrm{w} / \mathrm{v}$ could be explained by the fact that the positive surface charge on CS NPs was mainly due to the residual amine groups of CS that were not interacted with the TPP ions. Thus, the zeta potential of NPs was increased linearly with increasing the CS concentration as more nonneutralised $-\mathrm{NH}_{3}{ }^{+}$groups were present on the surface of NPs. However, in case of 0.4 and $0.5 \% \mathrm{w} / \mathrm{v}$ CS solutions, a slight decrease in surface charge was expected to be caused by the less $\mathrm{H}^{+}$ ions liberated from the acetic acid $\left(\mathrm{CH}_{3} \mathrm{COO}^{-} \mathrm{H}^{+}\right)$which had been fully used up to protonate the $-\mathrm{NH}_{2}$ groups on CS. Moreover, TPP solution donated some extent of the hydroxide ions, besides phosphoric ions [26]. Therefore, when the liberated $\mathrm{H}^{+}$ions from the acetic acid were not sufficient to neutralize the hydroxide ions $\left(-\mathrm{OH}^{-}\right)$of TPP, the $-\mathrm{OH}^{-}$ions would then tend to ionically crosslink with the protonated $-\mathrm{NH}_{3}{ }^{+}$groups of CS [28] and thus reduced the zeta potential of CS NPs [27].

3.3.2. EE of HC-Loaded CS NPs. Table 1 also represents the effect of CS concentrations on the EE of HC-loaded CS NPs. It was found that the EE of HC-loaded CS NPs was slightly (not significantly, one-way ANOVA, $P>0.05$ ) improved from $86 \pm 3$ to $88 \pm 4 \%$ with increasing the CS concentration from $0.1 \%$ to $0.3 \% \mathrm{w} / \mathrm{v}$. The increase in EE may be due to the increase in $-\mathrm{NH}_{3}{ }^{+}$groups on the particle surface. The increased $-\mathrm{NH}_{3}{ }^{+}$groups would facilitate a stronger electrostatic entanglement between $\mathrm{HC}$ and CS [29] and thus promote the entrapment of HC. In contrary to that, the high EE of HC-loaded CS NPs for 0.4 to $0.5 \% \mathrm{w} / \mathrm{v}$ CS solutions was also observed despite their low zeta potential values. This could be explained by the fact that increase in CS concentration will increase the viscosity of the CS solution and prevent the $\mathrm{HC}$ molecules from leaving the nanomatrix. This therefore, enhances the EE [30]. These results were also in accordance with some previously reported results $[29,31]$. In this study, the results of EE obtained from both U.V/Visspectrophotometer and RP-HPLC were also compared as shown in Table 1. The results from both methods were similar and comparable.

3.4. FT-IR Analysis. FT-IR spectra of LMwt CS, HC, and HC-loaded LMwt CS NPs are presented in Figure 3. The results showed that the intense characteristic peaks of CS (Figure 3(a)) were appearing at $3430 \mathrm{~cm}^{-1}$ (-OH stretching), 2914 and $2882 \mathrm{~cm}^{-1}$ (-CH stretching), $1656,1595 \mathrm{~cm}^{-1}$ (- $\mathrm{NH}_{2}$ stretching), 1324, $1256 \mathrm{~cm}^{-1}$ (C-N stretching), and $1076 \mathrm{~cm}^{-1}$ (C-O-C stretching). Figure $3(\mathrm{~b})$ shows that the characteristics peaks of $\mathrm{HC}$ were appearing at $3435 \mathrm{~cm}^{-1}$ (-OH stretching), 2972, $2931 \mathrm{~cm}^{-1}$ (C-H stretching), and $1714 \mathrm{~cm}^{-1}$ ( $-\mathrm{C}=\mathrm{O}$ bending). For HC-loaded CS NPs (Figure $3(\mathrm{c})$ ), the intense peaks of $3403 \mathrm{~cm}^{-1}$ was become broader, indicating that hydrogen bonding has been enhanced between the $-\mathrm{OH}$ bending groups of $\mathrm{HC}$ at $3435 \mathrm{~cm}^{-1}$ and $\mathrm{CS}$ at $3430 \mathrm{~cm}^{-1}$ [32]. The amide I and amide II bending vibrations in CS spectra shifted from $1656 \mathrm{~cm}^{-1}$ and $1595 \mathrm{~cm}^{-1}$ to $1636 \mathrm{~cm}^{-1}$ and $1535 \mathrm{~cm}^{-1}$, respectively, which indicated that some interaction has occurred between $-\mathrm{NH}_{3}{ }^{+}$groups of CS with TPP and HC within HC-loaded

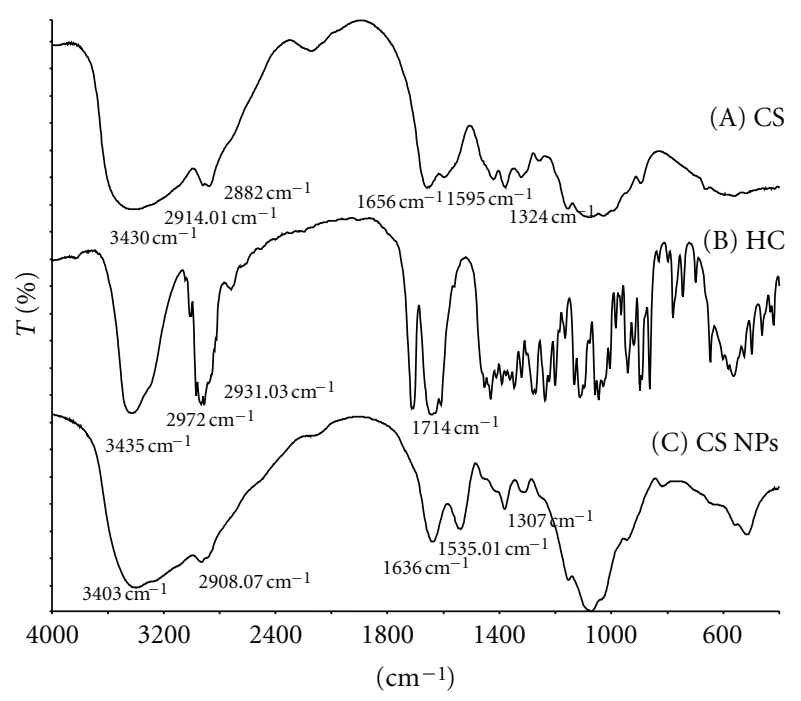

Figure 3: FT-IR spectra of (a) LMwt CS (b) pure HC and (c) HCloaded LMwt CS NPs (CS 0.2\% w/v, HC $1 \mathrm{mg} / \mathrm{mL}$, TPP $1 \mathrm{mg} / \mathrm{mL}$ ).

CS NPs spectra [33]. The peak at $1324 \mathrm{~cm}^{-1}$ (C-N bending) in CS spectra shifted to $1307 \mathrm{~cm}^{-1}$ in HC-loaded CS NPs spectra (Figure 3(c)). This indicated interaction between $-\mathrm{C}=\mathrm{O}$ group of $\mathrm{HC}$ and primary amide group of CS. The results may indicate that $\mathrm{HC}$ was successfully loaded into CS NPs.

3.5. Morphology of HC-Loaded CS NPs. Morphology of different HC-loaded CS NPs was investigated by using a TEM. The morphology of HC-loaded CS nanoparticles was found to be influenced by the molecular weight and type of chitosan used. LMwt CS produced a more spherical particle compared to HMwt CS as depicted by Figures 4(a) and 4(b), respectively. Contrary to that, TMC produced an elongated particle (Figure 4(c)). The mean particle size was observed to be decreased in the order of HMwt to LMwt CS NPs and TMC NPs. Morphology of LMwt CS NPs was also studied at different $\mathrm{pH}$ of CS solutions. It was observed that the LMwt HC-loaded CS NPs were spherical at a low $\mathrm{pH}(\mathrm{pH} 3)$ as depicted from Figure $4(\mathrm{c})$ and more of irregular shapes at higher pHs (Figures $4(\mathrm{~d})$ to $4(\mathrm{~g})$ ).

3.6. In-Vitro Drug Permeation of HC-Loaded LMW CS NPs. In-vitro cumulative drug permeation of $0.5 \%$ pure $\mathrm{HC}$ from aqueous and QV cream (served as positive controls) was investigated at different $\mathrm{pHs}$ of release media (4.0 and 7.4) as shown in Figures 5(a) and 5(b). The permeation of $\mathrm{HC}$ from HC-loaded CS NPs incorporated into both creams was also monitored as depicted by Figures 5(c) and 5(d). The results demonstrated that a higher $\mathrm{pH}$ of release media favored the permeation of HC from the positive controls as well as both creams containing HC-loaded CS NPs. A higher permeation of $\mathrm{HC}$ from formulations containing CS NPs at $\mathrm{pH} 7.4$ could be due to the porosity of CS NPs which was influenced by the $\mathrm{pH}$ of release media. The conversion of positively charged amino groups $\left(-\mathrm{NH}_{3}{ }^{+}\right)$of $\mathrm{CS}$ into the unionized state at 


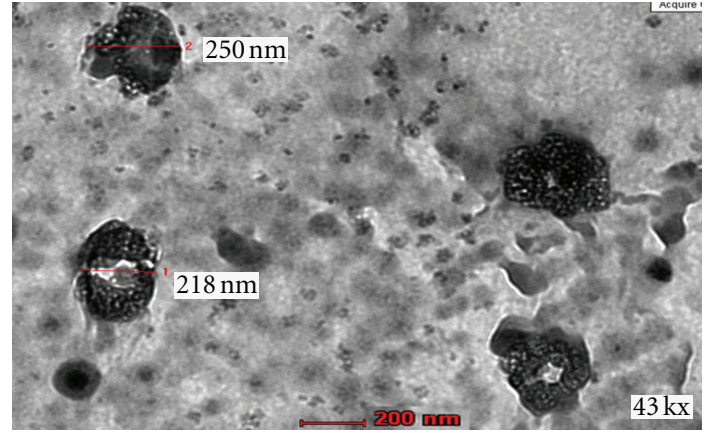

(a)

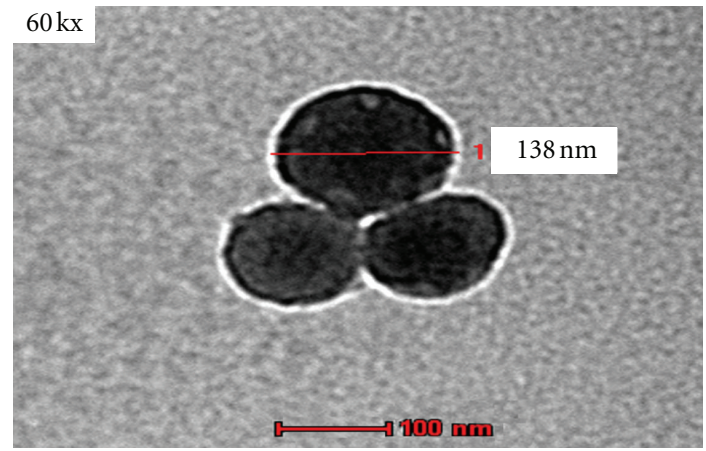

(c)

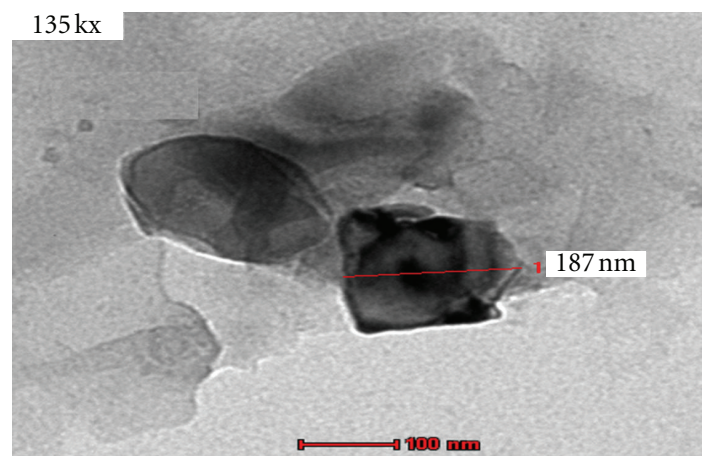

(e)

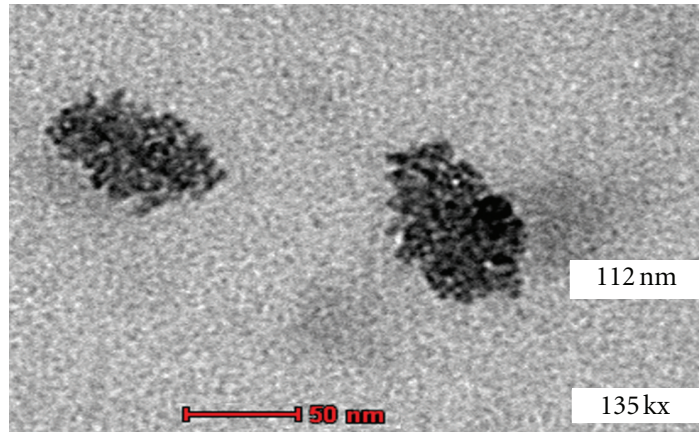

(b)

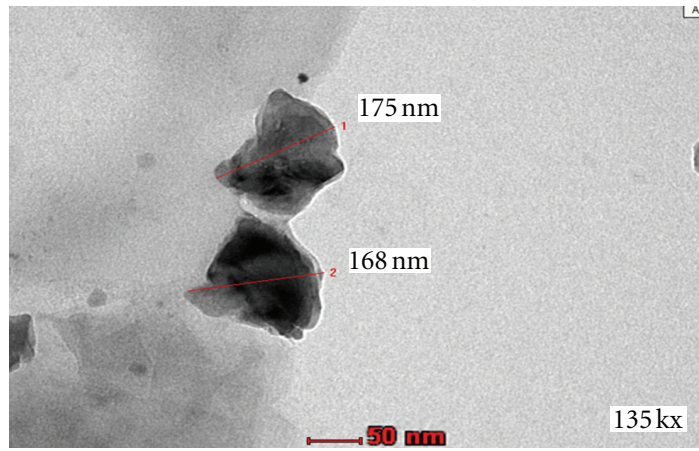

(d)

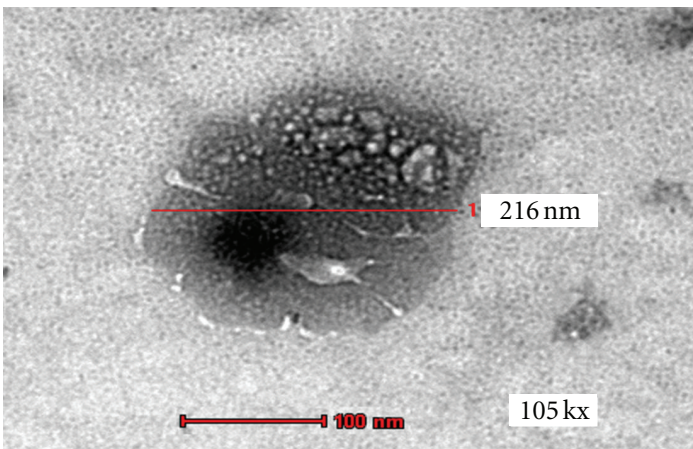

(f)

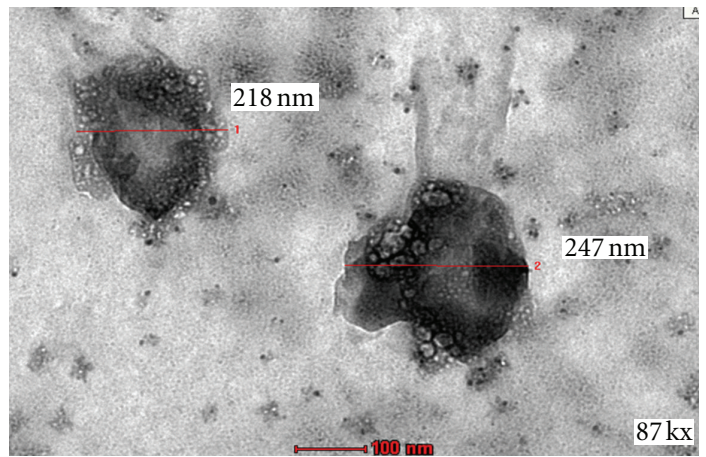

(g)

Figure 4: TEM images of HC-loaded HMwt CS NPs (a) LMwt CS NPs (b) TMC NPs (c) at pH 3.0. Morphology of LMwt CS NPs at pH 4 (d), 5 (e), 6 (f), and 7 (g) of CS solution. NPs were prepared from $0.2 \% \mathrm{w} / \mathrm{v} \mathrm{CS,} 1 \mathrm{mg} / \mathrm{mL}$ TPP and $1 \mathrm{mg} / \mathrm{mL} \mathrm{HC,} n=3$. 

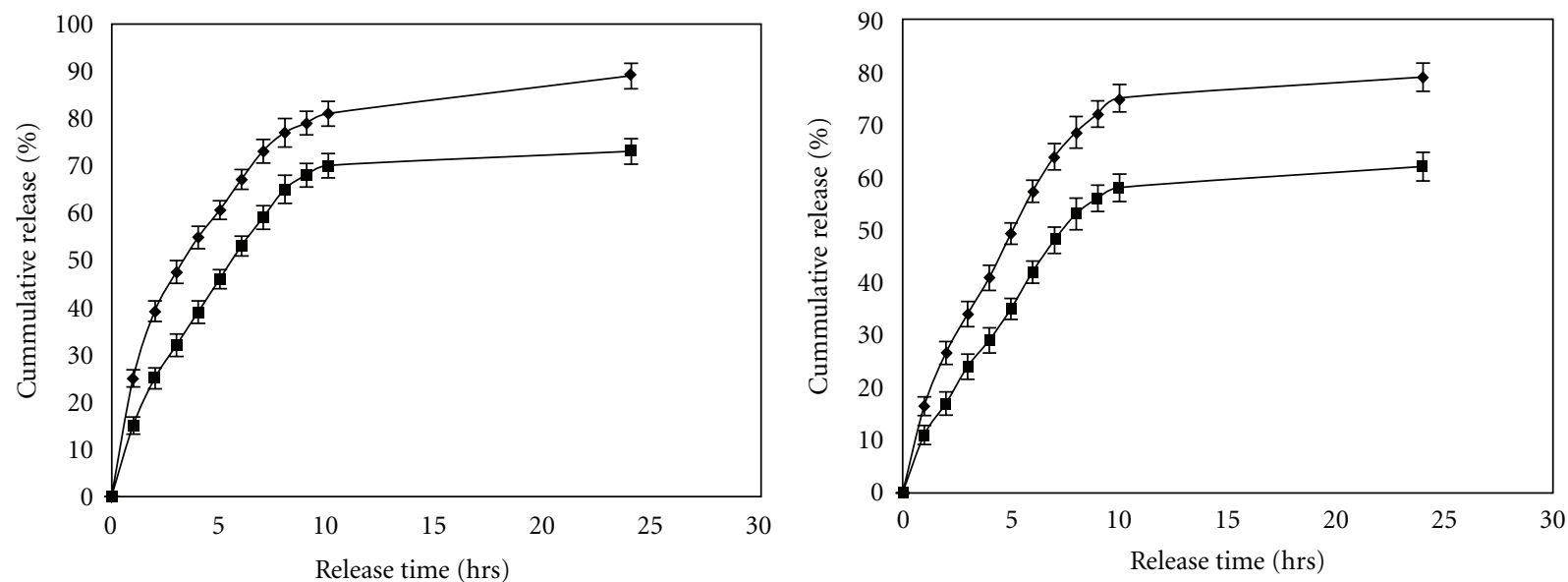

$\mathrm{QV}$ cream (control positive)
$\therefore-\mathrm{pH}=7.4$

Aqueous cream (control positive)

$\rightarrow \mathrm{pH}=7.4$

$\because \mathrm{pH}=4$

(a)

(b)

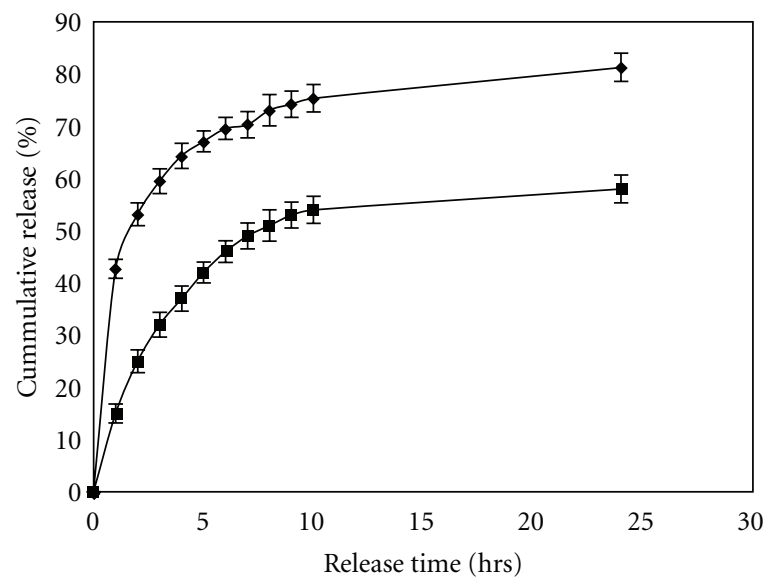

QV cream (HC-NPs)

$\rightarrow \mathrm{pH}=7.4$

$\rightarrow \mathrm{pH}=4$

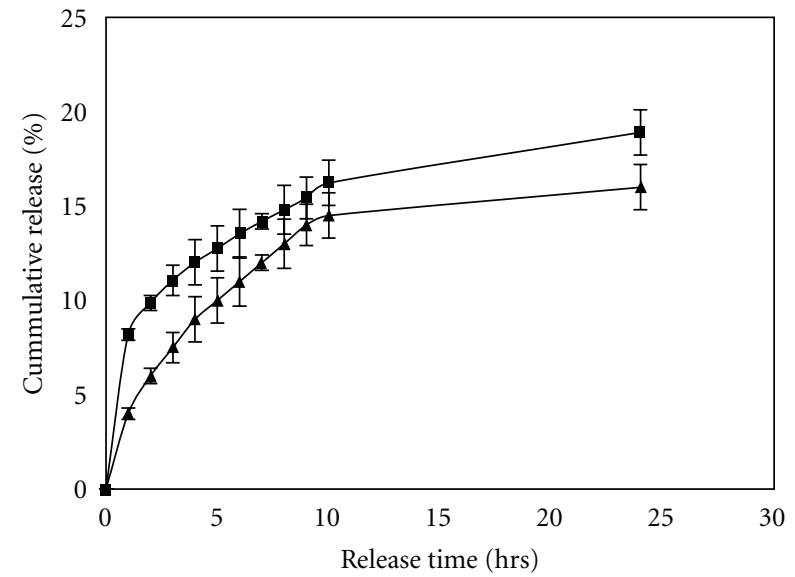

Aqueous cream (HC-NPs)

$\because \mathrm{pH}=7.4$

$\multimap \mathrm{pH}=4$

(c)

(d)

FIGURE 5: In-vitro cumulative permeation of HC from (a) QV cream (positive control), (b) aqueous cream (positive control), (c) QV cream containing HC-loaded LMwt CS NPs, and (d) aqueous cream containing HC-loaded LMwt CS NPs at pH 7.4 and 4.0 ( $n=3$ ).

a higher $\mathrm{pH}$ value resulted in the reduction of CS crosslinking extent with the counterion (TPP). As a result, the swelling of CS NPs might be increased [34] at higher $\mathrm{pH}$ value (7.4) as explained in Section 3.2. The swelling of CS NPs would facilitate the penetration of release media into the inner part of the polymer matrix and convert the glassy polymer to rubbery form. This subsequently facilitated the diffusion of HC molecules out from the CS NPs. The permeation of HC from positive controls was also found to be higher at a higher $\mathrm{pH}$ of release media. The release behavior was expected to be influenced by the $\mathrm{pKa}$ and $\mathrm{pH}$ values of the drug and release media.

Furthermore, the permeation of HC was higher from both creams without the addition of CS NPs. However, this was not observed for HC-loaded CS NPs in QV cream which had comparable cumulative drug release to the positive control at $\mathrm{pH}$ 7.4. The data also explored that the HC permeation from QV cream for both control and containing CS NPs was more efficient than aqueous cream as shown in Figure 5. The reason for a higher release of HC from QV cream than the aqueous cream was expected to be associated to the hydrophobicity of the QV cream contents which contributed to the consistent diffusion and dissolution of $\mathrm{HC}$ molecules. Moreover, it was expected that a high $\mathrm{HC}$ release from $\mathrm{QV}$ cream was attributed to the presence of glycerol $\left(\mathrm{CH}_{2} \mathrm{OH}-\right.$ $\mathrm{CHOH}-\mathrm{CH}_{2} \mathrm{OH}$ ) which is viscous and hygroscopic in nature [35]. The hygroscopicity of glycerol could draw some release medium (PBS:Ethanol) and facilitated the penetration of 


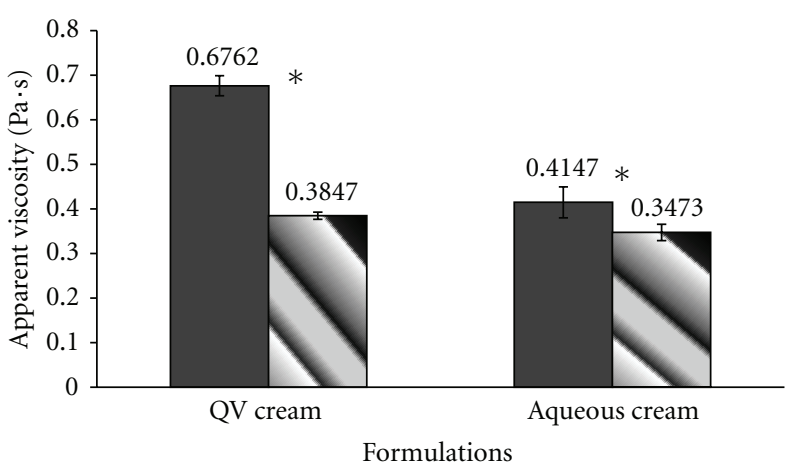

- Before adding NPs \& After adding NPs

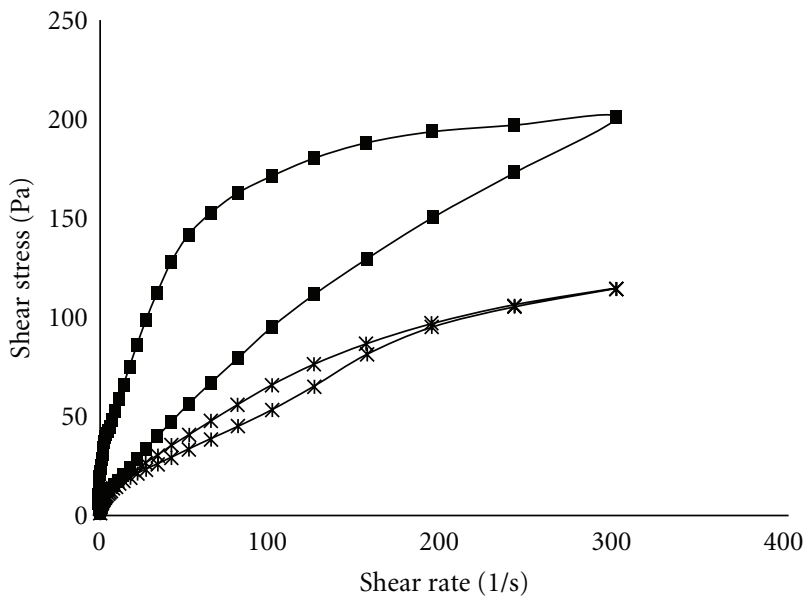

$\rightarrow$ Without NPs

$\rightarrow$ With NPs

(a)

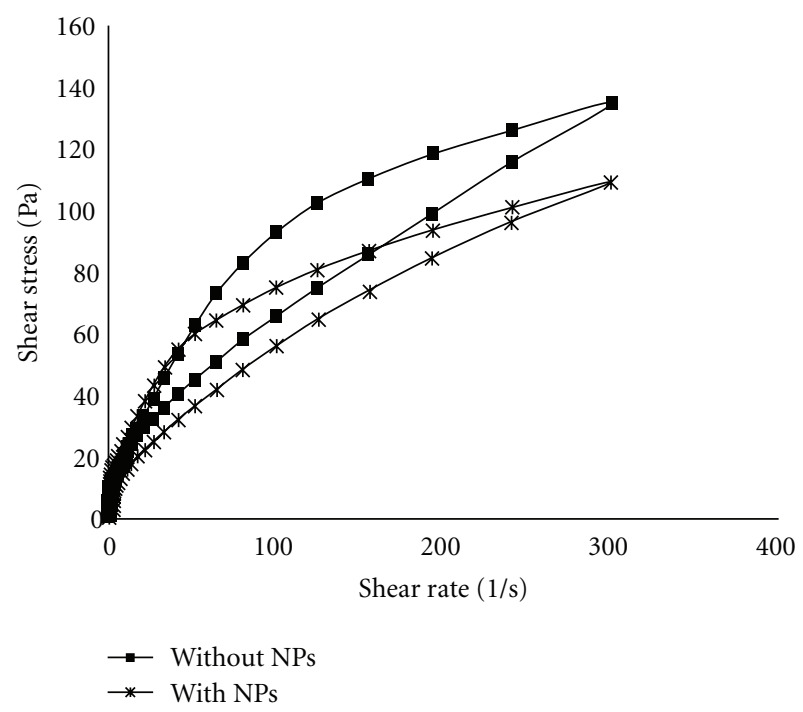

(c)

FIGURE 6: Comparative results of (a) apparent viscosities of QV cream and aqueous cream before and after loading with HC-loaded LMwt CS NPs (b) rheogram of QV cream before and after loading with HC-loaded CS NPs and (c) rheogram of aqueous cream before and after loading with HC-loaded CS NPs. NPs were prepared from $0.2 \% \mathrm{w} / \mathrm{v}$ CS, $1 \mathrm{mg} / \mathrm{mL} \mathrm{HC}$ and $1 \mathrm{mg} / \mathrm{mL}$ TPP, $n=3$.

medium inside the polymer matrix. This penetrated liquid could cause swelling of polymer matrix and led to the rapid diffusion and release of entrapped HC. Besides, a lower HC release from the aqueous cream could be due to the hydrophobic nature of $\mathrm{HC}$ which could not diffuse easily in the aqueous vehicle and perhaps due to the fact that the CS NPs remained intact in the aqueous vehicles [36]. Despite low permeation of HC from the CS NPs in aqueous cream, it is anticipated that this formulation may serve as a sustained release delivery system for $\mathrm{HC}$ which thought to be necessary to reduce side effects of $\mathrm{HC}$.

3.7. Rheological Characterization. To improve the release performance and stability of formulation, the rheological status is a very important physical parameter $[37,38]$. The apparent viscosity of the QV cream and aqueous cream before and after adding HC-loaded CS NPs is presented in Figure 6(a). The data illustrated that the apparent viscosities $\left(300 \mathrm{~s}^{-1}\right)$ of QV cream and aqueous cream before adding NPs were higher than the apparent viscosities after adding NPs into the formulations. Moreover, the apparent viscosity of QV cream was higher $(0.6762 \mathrm{~Pa} \cdot \mathrm{s})$ than the aqueous cream $(0.4147 \mathrm{~Pa} \cdot \mathrm{s})$. QV cream had also shown a significant reduction in the apparent viscosity from $0.6762 \mathrm{~Pa} \cdot \mathrm{s}$ to $0.3847 \mathrm{~Pa} \cdot \mathrm{s}$ after adding NPs $(P<0.05$, paired $t$-test $)$. From the rheogram of QV cream and aqueous cream (Figures 6(b) and 6(c)), it was observed that both creams showed shear rate dependency behavior or pseudoplastic flow. The decrease in 
the apparent viscosity with increasing shear rate was expected to be due to the structural breakdown of bonds which held the particles together [39]. This resulted in the formation of clusters or aggregates of droplets and finally led to the sharp reduction of the apparent viscosity [40].

\section{Conclusions}

In present research, the successful loading of HC into CS NPs via ionic-gelation method was confirmed by FT-IR analysis. It was demonstrated that the particle size, zeta potential, and EE of HC-loaded CS NPs were influenced by the molecular weight and type of CS as well as the concentration and $\mathrm{pH}$ of CS solution. The swelling behavior of HMwt, LMwt, and TMC NPs was also found to be modulated by the $\mathrm{pH}$ of incubating media. The resulted HC-loaded CS NPs were observed to be nonspherical and were influenced by the $\mathrm{pH}$ of CS solution. The findings suggested that the permeation of $\mathrm{HC}$ from the QV cream was more efficient than the aqueous cream and more profound at $\mathrm{pH} 7.4$ than 4.0. Despite low permeation of $\mathrm{HC}$ from CS NPs in aqueous cream, this formulation was considered to be a good candidate as a sustained release drug delivery system for HC. These findings therefore suggested that HC-loaded CS NPs held a promising potential as a potential delivery system for anti-inflammatory moieties which could improve drug efficacy and reduce related side effects.

\section{Conflict of Interests}

The authors declare that they have no conflict of interests.

\section{Acknowledgments}

Authors gratefully acknowledge the Ministry of Higher Education, Malaysia and Universiti Kebangsaan, Malaysia, for funding and support this research project. This research was funded by Arus Perdana Grant (UKM-AP-TKP-09-2010) and Fundamental Research Grant Scheme (UKM-FARMASI07-FRGS0015-2010).

\section{References}

[1] A. K. C. Leung and K. A. Barber, "Managing childhood atopic dermatitis," Advances in Therapy, vol. 20, no. 3, pp. 129-137, 2003.

[2] T. Zuberbier, S. J. Orlow, A. S. Paller et al., "Patient perspectives on the management of atopic dermatitis," Journal of Allergy and Clinical Immunology, vol. 118, no. 1, pp. 226-232, 2006.

[3] C. S. Maia, W. Mehnert, and M. Schäfer-Korting, "Solid lipid nanoparticles as drug carriers for topical glucocorticoids," International Journal of Pharmaceutics, vol. 196, no. 2, pp. 165$167,2000$.

[4] J. Hughes and M. Rustin, "Corticosteroids," Clinics in Dermatology, vol. 15, no. 5, pp. 715-721, 1997.

[5] S. Wiedersberg, C. S. Leopold, and R. H. Guy, "Bioavailability and bioequivalence of topical glucocorticoids," European Journal of Pharmaceutics and Biopharmaceutics, vol. 68, no. 3, pp. 453-466, 2008.
[6] E. O. Gilbertson, M. C. Spellman, D. J. Piacquadio, and M. I. Mulford, "Super potent topical corticosteroid use associated with adrenal suppression: clinical considerations," Journal of the American Academy of Dermatology, vol. 38, no. 2, pp. 318321, 1998.

[7] H. Schäcke, W. D. Döcke, and K. Asadullah, "Mechanisms involved in the side effects of glucocorticoids," Pharmacology and Therapeutics, vol. 96, no. 1, pp. 23-43, 2002.

[8] J. Y. Fang, Y. L. Leu, Y. Y. Wang, and Y. H. Tsai, "In vitro topical application and in vivo pharmacodynamic evaluation of nonivamide hydrogels using Wistar rat as an animal model," European Journal of Pharmaceutical Sciences, vol. 15, no. 5, pp. 417-423, 2002.

[9] K. S. Soppimath, T. M. Aminabhavi, A. R. Kulkarni, and W. E. Rudzinski, "Biodegradable polymeric nanoparticles as drug delivery devices," Journal of Controlled Release, vol. 70, no. 1-2, pp. 1-20, 2001.

[10] Y. Wu, W. Yang, C. Wang, J. Hu, and S. Fu, "Chitosan nanoparticles as a novel delivery system for ammonium glycyrrhizinate," International Journal of Pharmaceutics, vol. 295, no. 1-2, pp. 235-245, 2005.

[11] M. Schäfer-Korting, W. Mehnert, and H. C. Korting, "Lipid nanoparticles for improved topical application of drugs for skin diseases," Advanced Drug Delivery Reviews, vol. 59, no. 6, pp. 427-443, 2007.

[12] J. Davda and V. Labhasetwar, "Characterization of nanoparticle uptake by endothelial cells," International Journal of Pharmaceutics, vol. 233, no. 1-2, pp. 51-59, 2002.

[13] J. Panyam and V. Labhasetwar, "Biodegradable nanoparticles for drug and gene delivery to cells and tissue," Advanced Drug Delivery Reviews, vol. 55, no. 3, pp. 329-347, 2003.

[14] K. A. Janes, P. Calvo, and M. J. Alonso, "Polysaccharide colloidal particles as delivery systems for macromolecules," Advanced Drug Delivery Reviews, vol. 47, no. 1, pp. 83-97, 2001.

[15] R. Hejazi and M. Amiji, "Chitosan-based gastrointestinal delivery systems," Journal of Controlled Release, vol. 89, no. 2, pp. 151-165, 2003.

[16] K. Huanbutta, M. Luangtana-anan, P. Sriamornsak, S. Limmatvapirat, S. Puttipipatkhachorn, and J. Nunthanid, "Factor affecting preparations of chitosan microcapsules for colonic drug delivery," Journal of Metals, Materials and Minerals, vol. 18, pp. 79-83, 2008.

[17] M. Rinaudo, "Chitin and chitosan: properties and applications," Progress in Polymer Science, vol. 31, no. 7, pp. 603-632, 2006.

[18] P. Calvo, C. Remuñán-López, J. L. Vila-Jato, and M. J. Alonso, "Novel hydrophilic chitosan-polyethylene oxide nanoparticles as protein carriers," Journal of Applied Polymer Science, vol. 63, no. 1, pp. 125-132, 1997.

[19] S. Papadimitriou, D. Bikiaris, K. Avgoustakis, E. Karavas, and M. Georgarakis, "Chitosan nanoparticles loaded with dorzolamide and pramipexole," Carbohydrate Polymers, vol. 73, no. 1, pp. 44-54, 2008.

[20] Z. Z. Dai, J. B. Yin, S. F. Yan, T. Cao, J. Ma, and X. S. Chen, "Polyelectrolyte complexes based on chitosan and poly (Lglutamic acid)," Polymer International, vol. 56, no. 9, pp. 11221127, 2007.

[21] M. R. Avadi, A. M. M. Sadeghi, N. Mohammadpour et al., "Preparation and characterization of insulin nanoparticles using chitosan and Arabic gum with ionic gelation method," Nanomedicine, vol. 6, no. 1, pp. e58-e63, 2010. 
[22] H. Katas, E. Cevher, and H. O. Alpar, "Preparation of polyethyleneimine incorporated poly(d,l-lactide-co-glycolide) nanoparticles by spontaneous emulsion diffusion method for small interfering RNA delivery," International Journal of Pharmaceutics, vol. 369, no. 1-2, pp. 144-154, 2009.

[23] T. H. Kim, Y. H. Park, K. J. Kim, and C. S. Cho, "Release of albumin from chitosan-coated pectin beads in vitro," International Journal of Pharmaceutics, vol. 250, no. 2, pp. 371-383, 2003.

[24] A. Vila, A. Sánchez, K. Janes et al., "Low molecular weight chitosan nanoparticles as new carriers for nasal vaccine delivery in mice," European Journal of Pharmaceutics and Biopharmaceutics, vol. 57, no. 1, pp. 123-131, 2004.

[25] Y. T. Wu and C. Grant, "Effect of chelation chemistry of sodium polyaspartate on the dissolution of calcite," Langmuir, vol. 18, no. 18, pp. 6813-6820, 2002.

[26] W. Fan, W. Yan, Z. Xu, and H. Ni, "Formation mechanism of monodisperse, low molecular weight chitosan nanoparticles by ionic gelation technique," Colloids and Surfaces B, vol. 90, pp. 21-27, 2012.

[27] G. Qun and W. Ajun, "Effects of molecular weight, degree of acetylation and ionic strength on surface tension of chitosan in dilute solution," Carbohydrate Polymers, vol. 64, no. 1, pp. 29-36, 2006.

[28] X. Z. Shu and K. J. Zhu, "The influence of multivalent phosphate structure on the properties of ionically cross-linked chitosan films for controlled drug release.," European Journal of Pharmaceutics and Biopharmaceutics, vol. 54, no. 2, pp. 235 243, 2002.

[29] Y. Luo, B. Zhang, W. H. Cheng, and Q. Wang, "Preparation, characterization and evaluation of selenite-loaded chitosan/ TPP nanoparticles with or without zein coating," Carbohydrate Polymers, vol. 82, no. 3, pp. 942-951, 2010.

[30] V. R. Sinha, A. K. Singla, S. Wadhawan et al., "Chitosan microspheres as a potential carrier for drugs," International Journal of Pharmaceutics, vol. 274, no. 1-2, pp. 1-33, 2004.

[31] B. Hu, C. Pan, Y. Sun et al., "Optimization of fabrication parameters to produce chitosan-tripolyphosphate nanoparticles for delivery of tea catechins," Journal of Agricultural and Food Chemistry, vol. 56, no. 16, pp. 7451-7458, 2008.

[32] Y. Wu, W. Yang, C. Wang, J. Hu, and S. Fu, "Chitosan nanoparticles as a novel delivery system for ammonium glycyrrhizinate," International Journal of Pharmaceutics, vol. 295, no. 1-2, pp. 235-245, 2005.

[33] Y. Xu and Y. Du, "Effect of molecular structure of chitosan on protein delivery properties of chitosan nanoparticles," International Journal of Pharmaceutics, vol. 250, no. 1, pp. 215226, 2003.

[34] X. Z. Shu and K. J. Zhu, "A novel approach to prepare tripolyphosphate/chitosan complex beads for controlled release drug delivery," International Journal of Pharmaceutics, vol. 201, no. 1, pp. 51-58, 2000.

[35] L. Leroux, Z. Hatim, M. Frèche, and J. L. Lacout, "Effects of various adjuvants (lactic acid, glycerol, and chitosan) on the injectability of a calcium phosphate cement," Bone, vol. 25, no. 1, pp. 31S-34S, 1999.

[36] Y. Zhao, M. Moddaresi, S. A. Jones, and M. B. Brown, "A dynamic topical hydrofluoroalkane foam to induce nanoparticle modification and drug release in situ," European Journal of Pharmaceutics and Biopharmaceutics, vol. 72, no. 3, pp. 521$528,2009$.

[37] I. F. Almeida and M. F. Bahia, "Evaluation of the physical stability of two oleogels," International Journal of Pharmaceutics, vol. 327, no. 1-2, pp. 73-77, 2006.
[38] A. Lippacher, R. H. Müller, and K. Mäder, "Preparation of semisolid drug carriers for topical application based on solid lipid nanoparticles," International Journal of Pharmaceutics, vol. 214, no. 1-2, pp. 9-12, 2001.

[39] D. R. Izidoro, A. P. Scheer, M. R. Sierakowski, and C. W. I. Haminiuk, "Influence of green banana pulp on the rheological behaviour and chemical characteristics of emulsions (mayonnaises)," Food Science and Technology, vol. 41, no. 6, pp. 10181028, 2008.

[40] Z. Long, M. M. Zhao, Q. Z. Zhao, B. Yang, and L. Y. Liu, "Effect of homogenization and storage time on surface and rheology properties of whipping cream," Food Chemistry, vol. 131, pp. 748-753, 2011. 

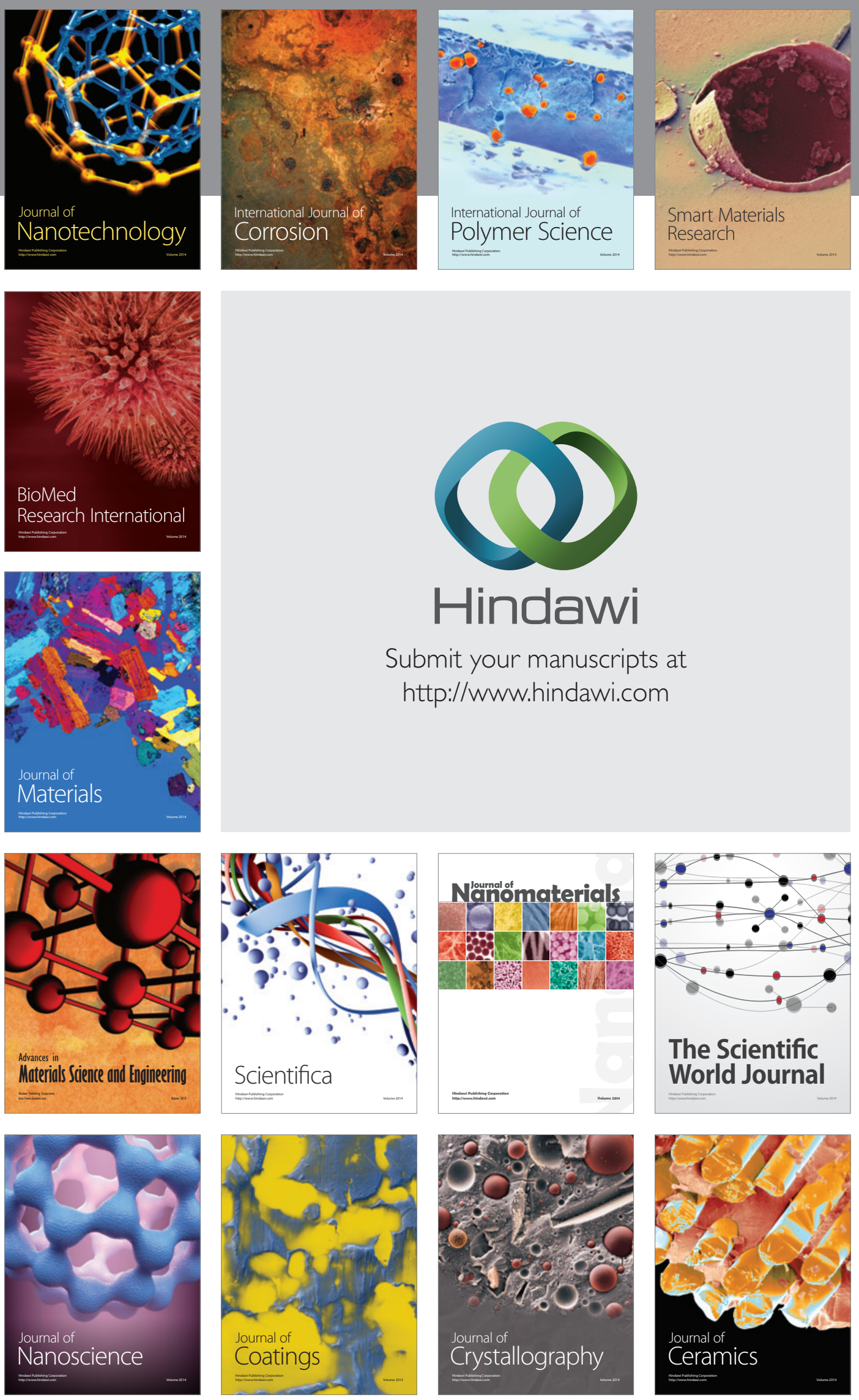

The Scientific World Journal

Submit your manuscripts at

http://www.hindawi.com

\section{World Journal}

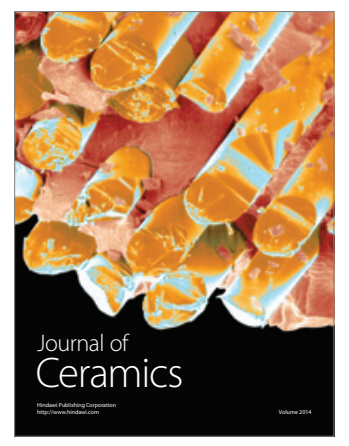

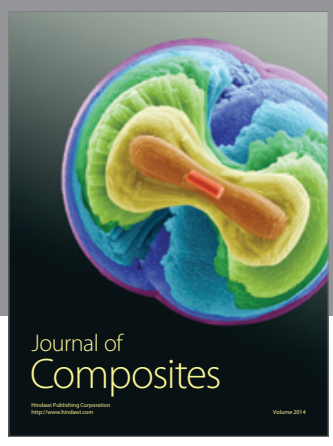
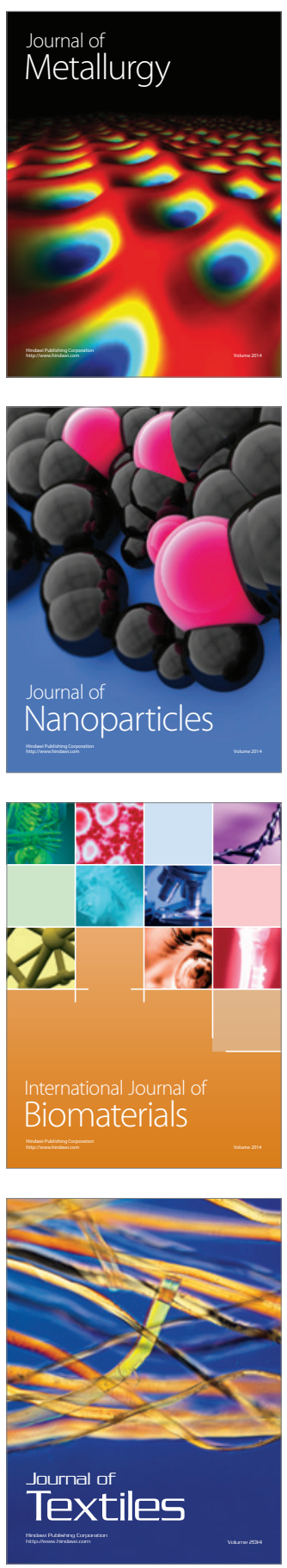